\title{
Robertsonian Translocation Between Chromosome Number 13 And 14 Associated With Recurrent Pregnancy Loss
}

\author{
Saba N, Verma Rk, Rani A, Kumar N, Singh U*, Faheem M ${ }^{* *}$ \\ ${ }^{I}$ Department Of Anatomy, King George's Medical University, U.P, Lucknow (India), \\ ${ }^{2}$ Department Of Obstetrics And Gynaecology, King George's Medical University, U.P, Lucknow (India)*, \\ ${ }^{3}$ Department Of Neurosurgery, King George's Medical University, U.P, Lucknow (India) ${ }^{* * *}$.
}

\begin{abstract}
introduction- Spontaneous miscarriage of a developing embryo may be due to the presence of rearrangements in parental chromosome set. The most common structural rearrangement involved in multiple abortions is chromosomal translocation. Robertsonian translocation shows the fusion of two acrocentric chromosomes, near the centromere region with loss of the short arms and it is found to be associated with recurrent pregnancy loss. Banding patterns has been the primary tool for the clinical assessment of patients to study chromosomal analysis from peripheral blood lymphocytes of cases with repeated pregnancy loss.

aims and objectives: Purpose of this study was to find out the Robertsonian translocations between chromosome 13 and 14 along with other chromosomal abnormalities in male and female partner having recurrent pregnancy loss.

materials and methods: 86 couples with history of repeated pregnancy loss were included during the period of July 2013 to June 2014 to study chromosomal analysis from peripheral blood lymphocytes. Karyogram were prepared according to standard cytogenetic methods using $G$-banding technique.

results: Total number of abnormal cases were 9 (10.46\%) with findings of abnormal karyogram in 3 (3.5\%) males and $6(7.0 \%)$ females. One case (1.16\%) of Robertsonian translocation, 1 case (1.16\%) of reciprocal translocation and 1 case (1.16\%) of sex chromosome mosaicism were observed in male partner, while 1 case (1.16\%) of Robertsonian translocation, 1 case (1.16\%) of reciprocal translocation, 3 cases (3.5\%) of mosaicism and 1 case of $(1.16 \%)$ of isochromosome were seen in females.

conclusion: Chromosomal translocations are common among structural chromosomal abnormalities and Robertsonian translocation between chromosome 13 and 14 is found to be an important cause in recurrent pregnancy loss.
\end{abstract}

\section{Introduction}

Recurrent miscarriages continue to be a challenging problem for both, the patients and the clinicians. Often cytogenetic studies have an important role in the evaluation of couples with a poor obstetric history ${ }^{[1]}$. Repeated pregnancy loss in first trimester is strongly associated with chromosomal abnormality in couples ${ }^{[2],[3]}$. Findings of the cytogenetic studies could provide valuable information for genetic counselling and allow monitoring of future pregnancies by prenatal diagnosis in couples with a common clinical problem defined as spontaneous pregnancy loss at less than 20-28 weeks gestation ${ }^{[4]}$. Spontaneous miscarriage of a developing embryo may be caused by the presence of rearrangements in parental chromosome set resulting in formation of gametes with unbalanced chromosomes like duplications or deletions by unequal crossing over during meiosis ${ }^{[5]}$. In 4-8\% of couples with recurrent pregnancy loss, at least one of the partners has chromosomal abnormalities ${ }^{[6]}$. The most common structural rearrangement involved in multiple abortions is Chromosomal translocation ${ }^{[5]}$. Translocation involves exchange of genetic material between two or more non-homologous chromosomes. Robertsonian translocation shows the fusion of two acrocentric chromosomes, near the centromere region with loss of the short arms. Carriers of these rearrangements are likely to produce unbalanced gametes resulting in abnormal offspring with unbalanced karyotype ${ }^{[7],}{ }^{[8]}$. Indeed, since the development of cytogenetic analysis in 1970s, banding patterns has been the primary tool for the clinical assessment of patients ${ }^{[9]}$. So we decided to study chromosomal analysis from peripheral blood lymphocytes of our cases with repeated pregnancy loss, according to standard cytogenetic methods using G-banding technique.

\section{Materials and methods}

In this descriptive study, a total of 86 couples with recurrent pregnancy loss were included during the period from July 2013 to June 2014. Karyogram was prepared from peripheral blood and cytogenetic analysis was conducted in cytogenetic laboratory of Department of Anatomy, King George's Medical University, Lucknow, India. Cases were enrolled in accordance with reproductive age and number of pregnancy losses. Couples not fulfilling these criteria were excluded from the study. 
Venous blood of $2 \mathrm{ml}$ was collected from both male and female partner in BD vacutainer sodium heparin vial. $0.5 \mathrm{ml}$ of blood was added in $5 \mathrm{ml}$ of culture media (RPMI1640) along with $0.1 \mathrm{ml}$ of phytohemagglutinin in a test tube which was kept for 3 days incubation at $37^{\circ} \mathrm{C}$ temperature in $\mathrm{CO}_{2}$ incubator with $85 \%$ humidity and $5 \%$ concentration of $\mathrm{CO}_{2}$ in slanting position.

Test tube was again incubated for 1 hour after adding 5 drops of colchicines $(0.1 \mu \mathrm{gm} / \mathrm{ml})$ and then centrifugation of test tube was done at $1000 \mathrm{rpm}$ for 10 minutes in a centrifuge machine. Supernatant was then discarded by pipetting of media and centrifugation was repeated 3-4 times with steps of re-suspension of cell button in $5 \mathrm{ml}$ of hypotonic solution (Potassium chloride+ Sodium citrate; 3:1) and $5 \mathrm{ml}$ of fixative (Methanol+ Acetic acid; 3:1) by dropping method on vortex. Fixative was added finally to make harvested cells ready for slide preparation by dropping method. Staining of slide was done by Giemsa stain after treatment with trypsin for better banding.

Slides were observed under a microscope (Olympus BX51) attached with computer and fields were photographed and karyogram was prepared with the help of cytovision software and also manually. Total 20 metaphase fields in the slide of a case were observed which were extended to fifty fields in case of suspected mosaicism. Karyotypes were reported as per International System for Human Cytogenetic Nomenclature (ISCN, 2009) guidelines.

\section{Results}

Total number of abnormal cases were $9(10.46 \%)$ with findings of abnormal karyogram in $3(3.5 \%)$ males and 6 (7.0\%) females (Table-1).

Table-1 Karyotype findings in male and female partner

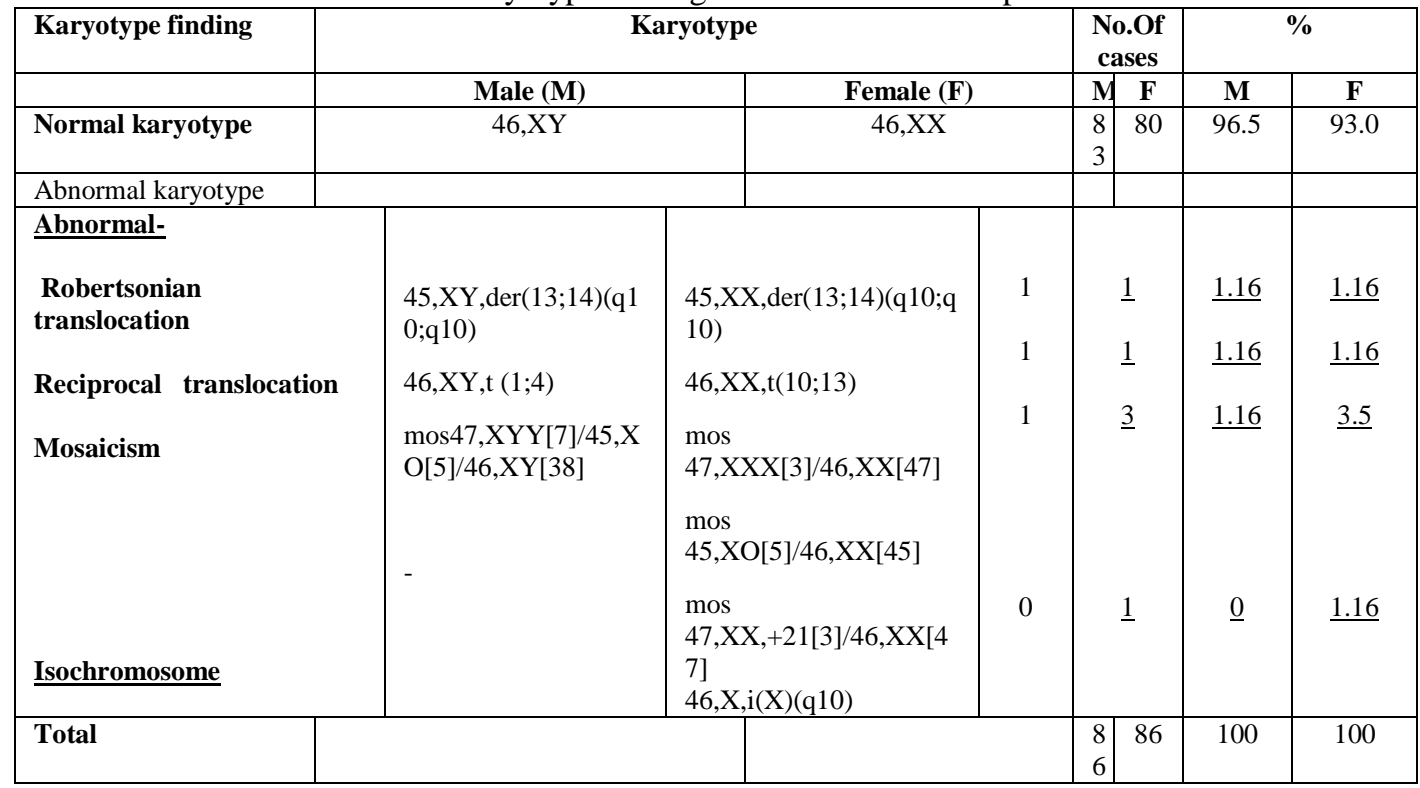

Normal karyotype was obtained in $83(96.5 \%)$ males and $80(93.0 \%)$ females. One case $(1.16 \%)$ of 45,XY,der(13;14)(q10;q10) i.e Robertsonian translocation (figure 1), 1 case $(1.16 \%)$ of $46, X Y, t(1 ; 4)$ i.e reciprocal translocation and 1 case $(1.16 \%)$ of mos 47,XYY[7]/45,XO[5]/46,XY[38] i.e sex chromosome mosaicism were observed in male partner, while a case $(1.16 \%)$ of $45, X X, \operatorname{der}(13 ; 14)(\mathrm{q} 10 ; \mathrm{q} 10)$ i.e Robertsonian translocation (figure 2), 1 case $(1.16 \%)$ of $46, \mathrm{XX}, \mathrm{t}(10 ; 13)$ i.e reciprocal translocation, 3 cases $(3.5 \%)$ of mos 47,XXX[3]/46,XX[47], mos 45,XO[5]/46,XX[45], mos 47,XX,+21[3]/46,XX[47] i.e mosaicism and 1 case of $(1.16 \%)$ of $46, X, i(X)(q 10)$ i.e isochromosome were seen in females (Table-1).

\section{Discussion}

A modest but clinically important proportion of spontaneous abortions is caused by a balanced chromosomal aberration in at-least one of the partners. This results from the production of gametes and embryos with unbalanced chromosome sets ${ }^{[10]}$. Total abnormal karyogram in present study were $9(10.46 \%)$ which was found to be parallel to various other studies as $9.04 \%^{[10]}, 9.06 \%{ }^{[11]}$ and $12.5 \%^{[12]}$. The range of prevalence in couples with defective reproductive success was $2.4 \%$ to $13.1 \%$ in which one of the partners was the carrier for a balanced chromosomal rearrangement, whereas this incidence was less than $0.55 \%$ in general population ${ }^{[13]}$. The variations in the size of the sample, the criteria used for ascertainment of cases and the technique of 
cytogenetic study have contributed to these wide differences between various studies. The male and female differences in finding of chromosomal aberrations were depicted in present study (Table-2).

Table-2 Chromosomal aberrations in males and females

\begin{tabular}{|c|c|c|c|}
\hline S. No. & Male & Female & Study \\
\hline 1 & $3.5 \%$ & $7.0 \%$ & Present study \\
\hline 2 & $5.0 \%$ & $8.0 \%$ & Niroumanesh et al (2011) ${ }^{[14]}$ \\
\hline 3 & $2.1 \%$ & $7.3 \%$ & Goncalves et al $(2014)^{[15]}$ \\
\hline 4 & $2.13 \%$ & $6.91 \%$ & Nazmy $(2008)^{[10]}$ \\
\hline 5 & $2.74 \%$ & $3.42 \%$ & El-Dahtory $(2011)^{[16]}$ \\
\hline
\end{tabular}

All these variable percent may be due to the fact that different populations vary in the incidence of carriers of chromosomal aberrations ${ }^{[17]}$. Also female to male ratio $2.1: 1^{[18]}$ goes in parallel with current findings, suggesting distribution of chromosomal anomalies in males and females near to the ratio of 1:2.

Robertsonian translocations constituted $2.32 \%$ of the cases with recurrent pregnancy loss and found to be higher than other studies. It was noted as $0.7 \%{ }^{[19]}, 0.8 \%{ }^{[20]}$ and $0.6 \%{ }^{[18]}$ in other studies which is less as compared to our study. In the present study this type of abnormality was $1.16 \%$ in both male and female, whereas in other study it was found to be $0.5 \%$ in males ${ }^{[21]}$ and $0.6 \%$ in females ${ }^{[15]}$. Robertsonian translocations present in our study were, $45, \mathrm{XX}, \operatorname{der}(13 ; 14)(\mathrm{q} 10 ; \mathrm{q} 10)$ in female and $45, \mathrm{XY}, \operatorname{der}(13 ; 14)(\mathrm{q} 10 ; \mathrm{q} 10)$ in male. This finding was also reported in other studies ${ }^{[21],[17],[10]}$, to focus the fact that der(13;14) can be frequently found in couples with reproductive failure. Apart from Robertsonian translocations other structural (reciprocal translocations \& isochromosome) and numerical abnormalities (mosaicism) were noted similar to studies conducted by various other Geneticists.

\section{Conclusion}

Among structural chromosomal abnormalities in recurrent pregnancy loss, translocations were very common with finding of Robertsonian translocation in chromosome 13 and 14. Thus, it can be concluded that Robertsonian translocation between chromosome number 13 and 14 should be specifically looked for while evaluating cases of recurrent pregnancy loss.

\section{References}

[1]. Iyer P, Wani L, Joshi S, Lakshmi J, Dalvi R, Chavan D, Das BR, Mandava S. Cytogenetic investigations in couples with repeated miscarriages and malformed children: report of a novel insertion. 2007; 14(3): 314-321.

[2]. Dubey S, Chowdhury MR, Prahlad B, Kumar V, Mathur R, Hamilton S, Kabra M, Menon PSN, Verma IC. Cytogenetic causes for recurrent spontaneous abortions- an experience of 742 couples (1484 cases). Indian Journal of Human Genetics. 2005; 11(2): 94-98.

[3]. Yuce H, Tekederell L, Elyas H. Cytogenetic results of recurrent spontaneous abortions in Turkey. Medical Science Monitor. 2007; 13(6): 286-289.

[4]. Goud TM, Al-Harassi SM, Al-Salmani KK, Al-Busaidy SM, Rajab A. Cytogenetic studies in couples with recurrent miscarriage in the sultanate of Oman. Reproductive Bio-Medicine Online. 2009; 18(3): 424-429.

[5]. Khedekar DN, Kesari GV, Kotwaliwale SS, Hattangdi SS. Cytogenetic study of leucocyte culture in multiple pregnancy loss in Mumbai. International Journal of Genetics. 2012; 4(2): 80-84.

[6]. Mustaqahamed S, Balachander V, Mohanadevi S, Arun M, Manikantan P, Sasikala K, Karthickkumar A, Silambuselvi, Sureshkumar S, Balamuralikrishnan B. Identification of cytogenetic alterations in infertile couples experiencing repeated spontaneous abortions- using Giemsa Trypsin Giemsa (GTG) banding. Scientific Research and Essays. 2011; 6(1): 182-186.

[7]. Purandare $\mathrm{H}$ and Chakravarty A. Human cytogenetic techniques and clinical applications. Bhilani publishing house, Mumbai, 2000; 182-203 \& 167

[8]. Cheng L and Zhang DY. Molecular Genetic Pathology in principles of clinical cytogenetics. Humana Press. Indianapolis, New York, 2008; 41-61.

[9]. Theisen A. Microarray-based comparative genomic hybridization (aCGH). Nature Education. 2008; 1(1): 45.

[10]. Nazmy NA. Cytogenetic studies of couples with reproductive failure in Alexandria, Egypt. Journal of Egypt Public Health Association, 2008; 83(3\&4): 255-271.

[11]. Chopade S, Chopade DK, Harde H. Impact of chromosomal heteromorphisms on recurrent miscarriages. Human Genet Embryol. 2012; $2: 101$.

[12]. Firoozabadi RD, Klantar S, Klantar S, Seyed-Hasani S, Ghasemi N, Asgharnia M, Sheikhha M. Cytogenetic analysis in couples with recurrent spontaneous abortion. Iranian Journal of Reproductive Medicine. 2006; 4(1): 13-17.

[13]. Nagaishi M, Yamamoto T, Linuma K. Chromosome abnormalities identified in 347 spontaneous abortions collected in Japan. J Obstet Gynaecol Res. 2004; 30: 237-41.

[14]. Niroumanesh S, Mehdipour P, Farajpour A, Darvish S. A cytogenetic study of couples with repeated spontaneous abortions. Annals of Saudi Medicine. 2011;31(1): 77-79.

[15]. Goncalves RO, Santos WVB, Sarno M, Cerqueira BAV, Goncalves MS, Costa OLN. Chromosomal abnormalities in couples with recurrent first trimester abortions. Rev. Bras. Ginecol. Obstet. 2014; 36(3): 113-117.

[16]. El-Dahtory FAM. Chromosomal abnormalities as a cause of recurrent abortions in Egypt. Indian Journal of Human Genetics. 2011; 17(2): 82-84.

[17]. Al-Hussain M, Al-Nuaim L, Abu Talib Z, Zaki OK. Cytogenetic study in cases with recurrent abortion in Saudi Arabia. Annals of Saudi Medicine. 2000; 20(3-4): 233-236.

[18]. Sheth FJ, Liehr T, Kumari P, Akinde R, Sheth HJ, Sheth JJ. Chromosomal abnormalities in couples with repeated fetal loss: an Indian retrospective study. Indian Journal of Human Genetics. 2013; 19(4): 415-422. 
[19]. Kochhar PK, Ghosh P. Reproductive outcome of couples with recurrent miscarriage and balanced chromosomal abnormalities. Journal of Obstetrics and Gynaecology Research. 2013; 39(1): 113-120.

[20]. Saxena SG, Desai K, Shewale L, Ranjan P, Saranath D. Chromosomal aberrations in 2000 couples of Indian ethnicity with reproductive failure. Reproductive BioMedicine Online. 2012; 25(2): 209-218.

[21]. Sider D, Wilson WG, Sudduth K, Atkin JF, Kelly TE. Cytogenetic studies in couples with recurrent pregnancy loss. Southern Medical Journal. 1988; 81(12): 1521-1524.

\section{IMAGES}

Figure 2: Karyogram of a female-45,XX,der(13;14)(q10;q10).
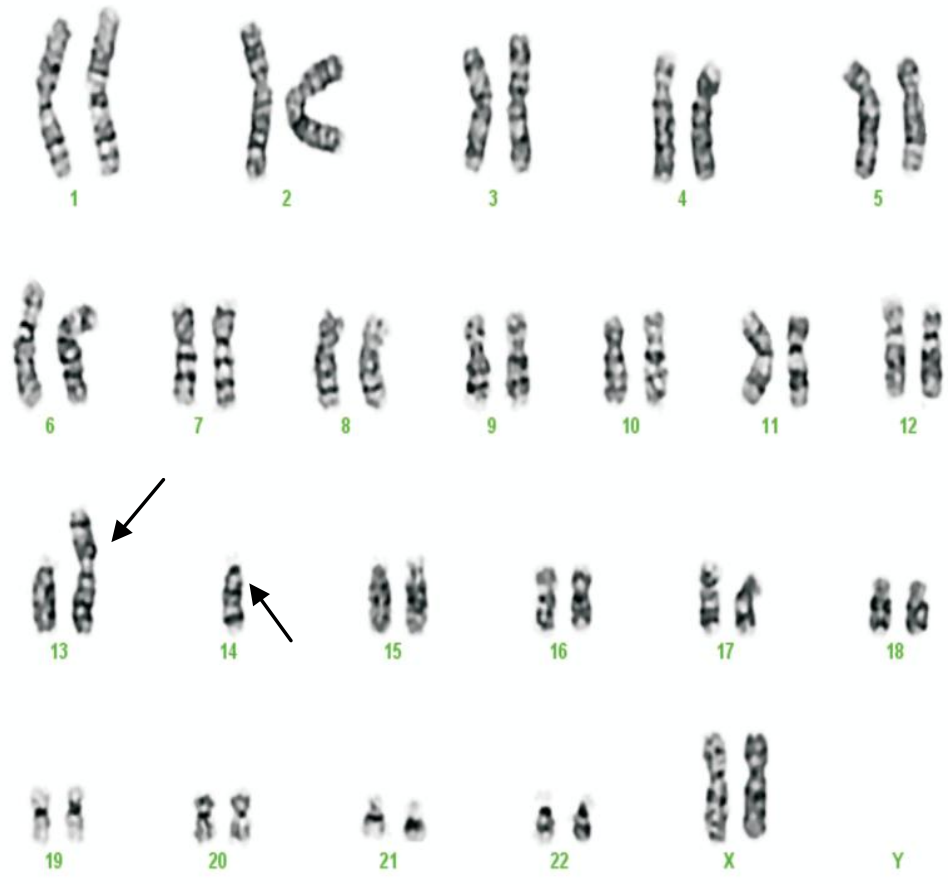

Figure 1: Karyogram of a male-46,XY,der(13;14)(q10;q10).
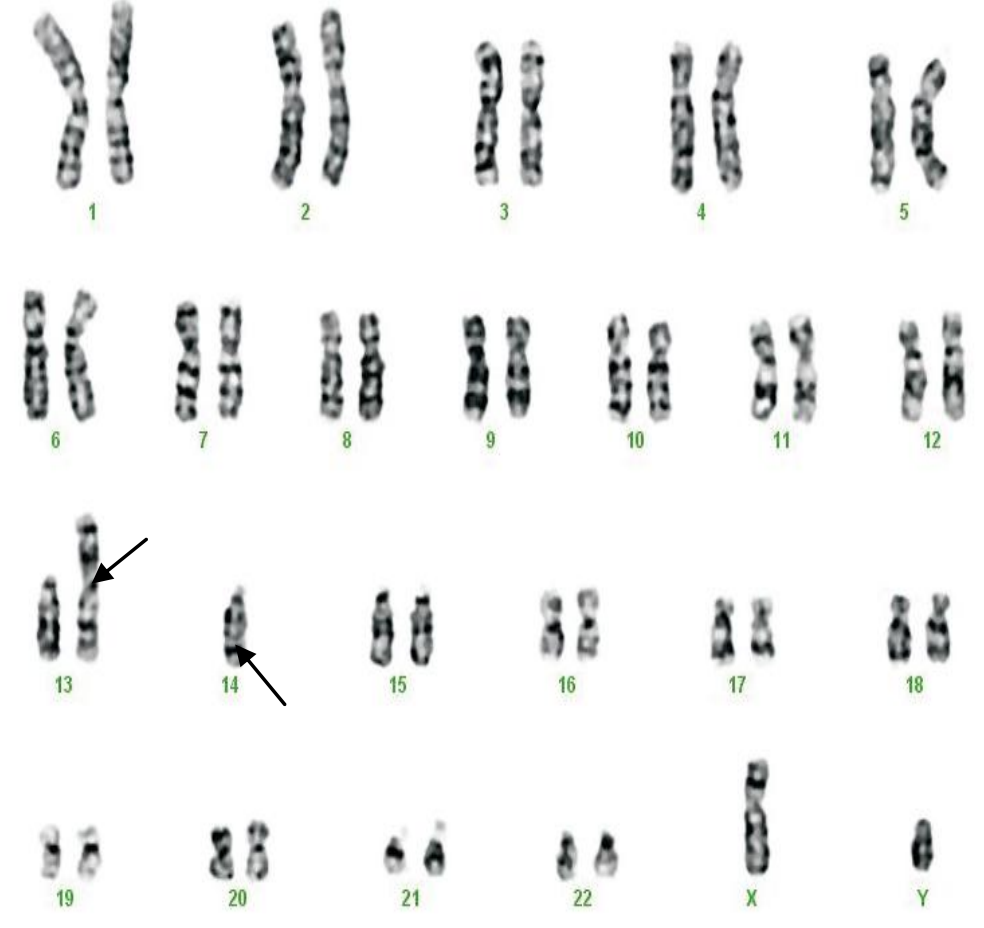